\title{
The technology of face recognition based on computer vision Miao Bin
}

\author{
Shandong Police College, shandong,jinan,250014
}

\begin{abstract}
Keywords: ARM9; hardware structure; software design; face recognition
\end{abstract}
\begin{abstract}
This paper presents a face recognition system design based on computer vision and the specific realization method, including the hardware structure, face recognition specific algorithm, drive, and software design and implementation of the application. The system uses ARM9 chip as the core, the built-in Linux operating system, a dual camera with external USB interface, combined with the application, the embedded 3D face recognition method combining with software and hardware technology is proposed.
\end{abstract}

\section{Introduction}

The identity authentication technology based on biological characteristics have rapid development in recent years, which, identity verification based on facial features is the most natural and direct mean, so the computer face recognition technology is one of the most active and challenging fields. Especially the superiority of the face recognition technology in non-con environment and situation without shocking the human being detected has already more than the identification method, like fingerprint and iris, and break the bottleneck of 2D face recognition, eliminates the influence of illumination, facial expression, and a series of factors. The technology of 3D face recognition has become a hot topic being studied by many scholars nowadays. With the continuous development of computer vision technology, face recognition technology based on computer vision is also in constant improvement.

\section{Overall design}

According to the characteristics of computer vision and face recognition technology and its application idea in the field of testing, this paper defines the general idea of face recognition technology based on computer vision. The whole process of embedded 3D computer vision face recognition is accomplished by a computer, the main work process is: face detection, facial feature location and face modeling and 3D face recognition.

\section{The design of system hardware}

The device selection, circuit design, sealing mode and system efficiency, accuracy, physical security and anti-attack ability has a direct relationship in face recognition technology based on computer vision, its structure as shown in figure 1.

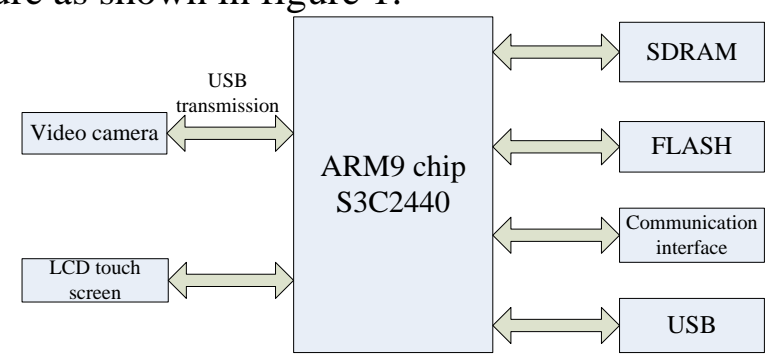

Figure1 the structure diagram of hardware

\section{Chip selection}

The microcontroller (Master CPU) is a key part of the face recognition system. These are the aspects have to be considered for selection, type of micro controller, bus width, performance, development tools, the support of peripheral device, cost, power consumption and cost performance. 
According to the above requirements, this paper chooses ARM9 chip to achieve the face recognition algorithm.

\section{Selection of cameras}

Image acquisition system adopts ANC company's Q888 type camera, CMOS photosensitive element, element pixels 1300000, transmission interface USB1.1. maximum resolution is 800*600DPI; 30fps maximum amount of frame can be supported, high clear glass sheet lens, automatic exposure and automatic gain control of built-in video compression unit, 2 to 8 times the camera compression ratio. Support for dynamic and static video acquisition. During image acquisition process, two camera is orthogonal as 90 degree, video is captured for the front and the side face. The collected pictures are sent to CPU, waiting for further processing.

\section{LCD display}

Computer visual display module is responsible for outputting the face model and the final recognition results, which is completed by LCD display. S3C2440 have built-in LCD controller, supporting 4 bit, 8 bit and 16 bit format color LCD of various types. Such as STN, TFT etc. Taking into account of the actual display effect and cost, this system use 3.5 320X240 LCD display of SHARP inch with TFT.

\section{The storage module}

The storage module is responsible for the storage of acquired image and processed data, to prepare for main CUP. The storage devices of the system is SDRAM, namely the synchronous DRAM. The biggest characteristic is that it can be synchronized with external working clock of CPU, SDRAM use the same clock as CPU in the system and the sovereign, if external working clock of CPU is $100 \mathrm{MHZ}$, the frequency sent to the memory is also $100 \mathrm{MHZ}$, which can improve the efficiency of the memory.

\section{The design of system software}

The software design mainly involves the following aspects: driver program design, face detection and face modeling program design and the program design of recognition algorithm. The driver program of this system includes the USB driver device, LCD driver, serial driver. Most of the hardware driver is completed by Linux, people just need to follow the corresponding hardware to set parameters and initialization operation. The program writing of related algorithm is the key for face recognition system, and an important factor for the success of $3 \mathrm{D}$ face recognition. In the software part, three key programs are paid more focus: face detection and feature point extraction program, face modeling program, 3D face recognition program.

\section{The design of face detection program}

The first step to realize face recognition, is to process accurate face detection and feature point extraction, which requires efficient face detection program. The proposed method is send image samples collected by the camera to the memory module, by image samples have been obtained to judge the face position, so as to select the appropriate face and extracts as the sample. Facial feature point extraction quality also has a direct impact on the 3D face image recognition effect. The following is program design idea for face detection. First of all, through the single frame picture transmitted by camera to identify the human eye coordinates (x1, y1) and (x2, y2), which can help to obtain coordinates of upper left and lower right vertex of square face indirectly, respectively (X1, Y1) and (X2, Y2), the detailed calculation method is shown as follows 


$$
\begin{aligned}
& \text { Width }_{\text {eyes }}=x_{2}-x_{1} \\
& \text { Width }_{\text {face }}=\text { Width }_{\text {eyes }} / R_{H} \\
& X_{1}=x_{1}-\left(\text { Width }_{\text {face }}-\text { Width }_{\text {eyes }}\right) / 2 \\
& X_{2}=X_{1}+\text { Width }_{\text {face }} \\
& \text { Height }_{\text {eyes }}=\left(y_{1}+y_{2}\right) / 2 \\
& \text { Height }_{\text {face }}=\text { Width }_{\text {face }} \\
& Y_{1}=\text { Height }_{\text {eves }}-\text { Height }_{\text {face }} / R_{v} \\
& Y_{2}=Y_{1}+\text { Height }_{\text {face }}
\end{aligned}
$$

In the formula, ${ }^{R_{H}}$ and ${ }^{R_{V}}$ are empirical constants, during the design process, its value is 2 and 3.5 respectively. So the regional coordinate of face in the original image can be obtained, the size is

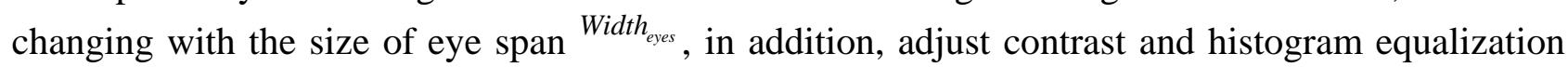
operation also need to be done to the picture, in order to improve the accuracy.

\section{Program design for face feature points extraction based on two-dimensional picture}

The extraction of the key feature points in face images, using AAM algorithm, this algorithm can effectively reduce the matching time. The specific method is as follows:

First, establish the shape expression formula:

$$
x=\bar{x}+Q_{s} c
$$

Facial texture expression formula:

$$
g=\bar{g}+Q_{g} c
$$

The $Q_{s}$ and $Q_{g}$ are the profile change pattern matrix and facial texture change matrix respectively, control parameters vector $c$ is used to control the change of the shape and texture, the regulation of c can help to obtain different shapes and facial texture, so as to synthesize different faces, the implementation process of AAM algorithm is: 1) the iterative search is done for every proportion, position, rotation angle of map 2) get the rough location. 3) continue to adjust the c value, and make the synthesized difference of face gray and image gray reaches the minimum value. 4) get fine mapping. 5) the location of each feature point is obtained by calculation.

\section{Face reconstruction program based on orthogonal projection}

According to the AAM algorithm to detect the two-dimensional coordinates of feature points, coordinating with the camera parameters, to transform the two-dimensional coordinates into 3D coordinates. Then according to the 3D coordinate to build a more complete face model. Here is specific algorithm to extract face 3D geometry features from the orthogonal (positive, side 90 degree orthogonal) photos.

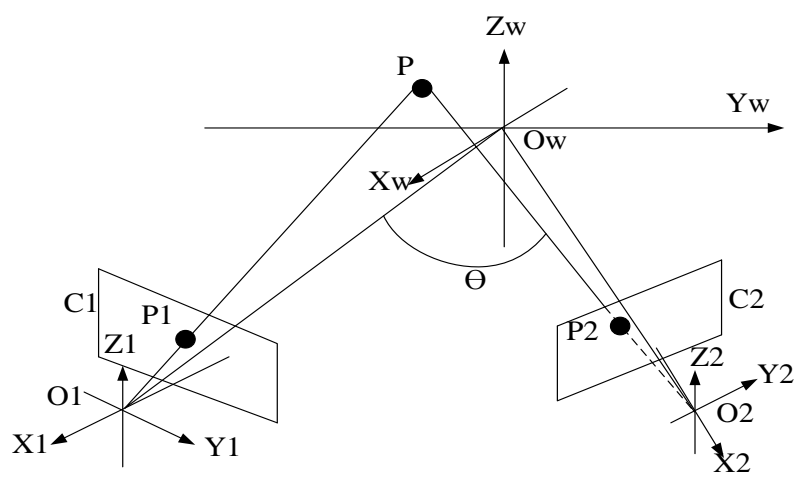

Figure 2 Schematic diagram of camera

Wherein, X1, X2 are the position of computer camera, have 90 degrees, images of the front and side face are acquired. $\mathrm{P}$ is the feature points in the image, $P 1$ and $P 2$ are camera imaging point of 90 degree angle, $P$ must be located on the intersection of 2 lines $O 1 P 1$ and $O 2 P 2$. When the photo is taken, the optical axis of camera ${ }^{C 1}$ axis have to be superposed with $\mathrm{Xo}$ axis of the face coordinate system, and the $\mathrm{Z} 1$ axis in the camera ${ }^{C 1}$ coordinates have to be paralleled to the $\mathrm{Zo}$ axis of face coordinates. Camera C2 is obtained after camera C1 rotated around the Z0 axis of $\theta$ degree angle in face coordinates. The coordinate of point $P$ in the face coordinate system is $(x, y, z)$, the distance 
between the optical center $O 1$ and $O w$ of camera $C 1$ is $D$, the camera parameters of $C 1$ and $C 2$ are the same and the focal length is $\mathrm{L}$, the coordinates of $P 1$ and $P 2$ in each camera image plane are

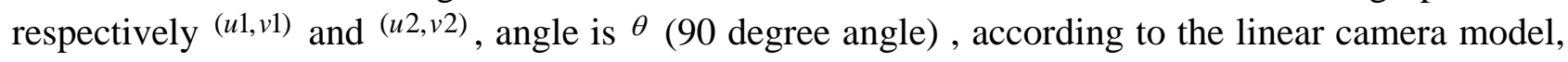
we can get the following conversion method:

$$
\left\{\begin{array}{c}
u 1=\frac{L}{D-X} Y \\
v 1=\frac{L}{D-X} Z \\
u 2=\frac{L}{D-(X \cos \theta+Y \sin \theta)}(Y \cos \theta-X \sin \theta) \\
v 2=\frac{L}{D-(X \cos \theta+Y \sin \theta)} Z
\end{array}\right.
$$

Only need to know the shooting distance $\mathrm{D}$ and camera focal length $L$, the coordinate of twodimensional feature points can be transformed into three-dimensional coordinate. Through the communication module to transfer the three-dimensional coordinate to CUP's memory, coupled with the OpenGL software under the Linux system to build 3D model.

Samples taken image as shown below:

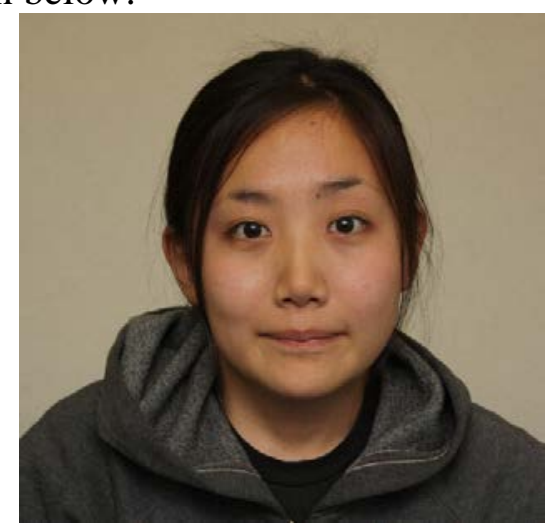

Figure 3 sample image

Below is a schematic diagram of the face model the established by method:

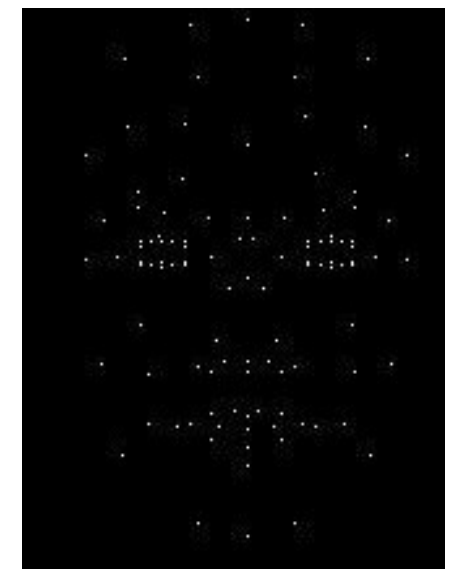

Figure 4: 3D face built by OpenGL according to the feature points 


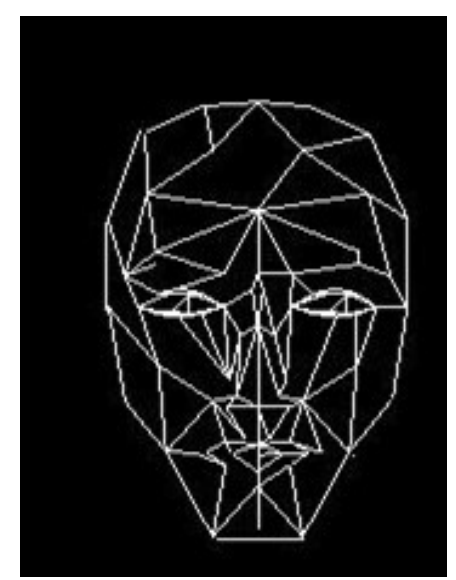

Figure 5: Triangle network topology structure of face

\section{Face recognition program design}

After the establishment of 3D face model, according to the three-dimensional information already stored in the storage module to recognize obtained 3D face information effectively. It can be compared according to the eigenvectors produced by face geometric features point data. Due to the restrictions of hardware in the extraction process, there may be some errors, so the similarity of eigenvectors have to be counted, which can increase the accuracy of recognition.

The formula for similarity calculation is as follows:

$$
\mathrm{S}=\sqrt{\left(t_{1}-p_{1}\right)^{2} k_{1}+\left(t_{2}-p_{2}\right)^{2} k_{2}+\ldots .+\left(t_{m}-p_{m}\right)^{2} k_{m}}
$$

Where $m$ is the dimension of eigenvectors; $t_{i}$ is i-th component of eigenvectors of sample need to be tested; $p_{i}$ is $\mathrm{i}$-th component of eigenvectors in storage library. $k_{i}$ is the weight of $\mathrm{i}$-th component of eigenvectors. The weight is determined by degree of importance of the facial features in anthropology. The similarity threshold $\theta$ is set to determine whether it is similar. When $S<\theta$, it is regarded as similar, recognition success, vice versa.

\section{Conclusions}

In recent years the face recognition system in computer vision due to its non-contact data acquisition, strong concealment, convenient, fast, easy to follow up and strong interactivity and other advantages, become one of the most popular research topics in pattern recognition and image processing. In this paper, combining with the actual project application, the design scheme of face recognition system based on computer vision is proposed, the implementation method of the hardware structure, drive and its application is provided, and the convenience for users to construct the face recognition system with different functions and regulate other computer system hardware structure is raised also.

\section{References}

[1] Guo Wenjie, Mu Zhichun. The face and ear multi-biological identity authentication based on feature fusion [J]. Journal of Tianjin University, 2009, 42 (7): 636-641.

[2]Ronald P.A survey on vision-based human action recognition[J].Image and Vision Computing,2010,28(6):976-990..

[3]Aggarwal J K,Ryoo M S.Human activity analysis:a review[J].ACM Computing Surveys,2011,43(3).

[4] Zhang Jie, Pan Zhongying, Lin Pingshi. USB interface design based on EZ-USB data acquisition system [J]. Electronic measurement technology, 2002, (04)

[5] Su Tao, Zhang Qiang, Wei Xiaopeng, Zhou Changjun. A novel face recognition method based on NMF [J]. Journal of system simulation, 2009, (12)

[6] Song Yu He Xiaoli, xian-bo he, dragon garden. Logistics vehicle scheduling algorithm based on cloud computing neural network study [J]. Computer simulation, 2012.4:367-370. 
[7] Wang, Mr Zhang xiu-fang li. Rararete: a parallelism matching algorithm based on Pete algorithm [J]. Journal of computer science, 2004, 31:112-114.

[8] Xiu-li zhang, GuanQi studied. A kind of expert system framework of Pete reasoning algorithm based on simplified design [J]. Journal of shenyang university of technology, 2008, 27 (5) : 100104.

[9] JiYing lan-ying li, hand, lei-lei zhang. Based on the genetic and tabu search hybrid algorithm [J]. Computer engineering and application, 2009, (20) : 81-83.

[10] Zhi-hui xiong, si-kun li, ji-hua Chen. Genetic algorithm and ant algorithm of dynamic fusion hardware/software partitioning [J]. Journal of software, 2005, 16 (4) : 503-512.

[11] Xiao-dong guo, liu jiren, Wen Hui. A hardware/software partitioning method based on genetic algorithm (ga). Journal of computer-aided design and graphics, 2001, 13 (1) : 24-27. 